\title{
Immunolocalization of the hepatocyte growth factor (HGF) system in the rat ovary and the anti-apoptotic effect of HGF in rat ovarian granulosa cells in vitro
}

\author{
Mehmet Uzumcu' ${ }^{2}$ Zui Pan², Yi Chu , Peter E Kuhn² and Rob Zachow ${ }^{1,2}$ \\ ${ }^{1}$ Department of Physiology and Biophysics, Robert Wood Johnson Medical School, University of Medicine and \\ Dentistry of New Jersey, Piscataway, New Jersey 08854, USA and ${ }^{2}$ Department of Animal Sciences, Rutgers \\ University, New Brunswick, New Jersey 08901, USA \\ Correspondence should be addressed to R Zachow; Email: zachowrj@umdnj.edu
}

\begin{abstract}
Hepatocyte growth factor (HGF) regulates granulosa cell (GC) steroidogenesis and suppresses apoptosis in non-ovarian cells. The hypothesis was thus developed that intraovarian HGF supports folliculogenesis by mediating steroidogenesis and suppressing apoptosis. To investigate the latter, the anti-apoptotic actions of HGF were tested in GCs and follicles isolated from immature rats. Results showed that HGF suppressed apoptosis in GC and follicle cultures as visualized using apoptosis indicator dye, YO-PRO-1. Immunohistochemistry was used to investigate the distribution of HGF, c-met, and HGF activator (HGFA) protein during folliculogenesis in equine chorionic gonadotropin (eCG)-primed rats. Immunoreactive HGF content was the greatest in GCs within preantral follicles. Following eCG, large antral follicles showed elevated HGF staining in theca and interstitial cells when compared with GCs. Intense c-met staining was observed in GCs within non-primed small preantral follicles; following eCG, the level of c-met was diminished in GCs, but increased within theca and interstitial cells. Theca, interstitium, and GCs in non-primed and primed ovaries contained HGFA. Following eCG, HGFA was more apparent in theca cells and the interstitium when compared to that in GCs within large antral follicles. The presence of HGF, c-met, and HGFA in preantral follicles would potentially enable the anti-apoptotic effects of HGF that were observed in vitro to occur in vivo. Advanced folliculogenesis led to a change in the cellular distribution of the HGF, c-met, and HGFA, suggesting that the ovarian HGF system is hormonally regulated in vivo.

Reproduction (2006) 132 291-299
\end{abstract}

\section{Introduction}

Interestingly, within the follicular cohort that is recruited to grow during each cycle of the reproductive life span, the overwhelming majority of follicles will not complete the course of folliculogenesis to fully mature and ovulate. Instead, all but the selected follicles undergo atresia. Apoptosis, especially within granulosa cells (GCs), is the cellular death mechanism that leads to follicular atresia. Therefore, over the span of reproductive life, the collective of recruited follicles that are not destined for selection comprise a massive unused source of gametes.

The precise signals, which determine follicular fate (ovulation vs atresia) are not known; however, several ligand-directed cellular mechanisms that promote either growth (cell survival) or atresia (apoptosis) have been identified. It appears that FSH, and the modulation of
FSH bioactivity by select intraovarian growth factors, can protect GC from apoptosis (Hussein 2005).

Hepatocyte growth factor (HGF) exerts autocrine and paracrine regulation of GC and theca cell growth and steroidogenesis (Liu et al. 1994, Parrott et al. 1994, Zachow et al. 1997, 2000, Lail-Trecker et al. 1998, Parrott \& Skinner 1998a,b, Osuga et al. 1999, Zachow \& Woolery 2002, Shimizu et al. 2003). Despite its welldocumented anti-apoptotic bioactivity in other cells (Lail-Trecker et al. 1998), the ability of HGF to control the onset of apoptosis in GC has not been previously reported.

Although HGF and its receptor (c-met) are expressed within the ovaries of numerous mammalian species (e.g., human, rat, pig, cow, sheep, and mouse) (Liu et al. 1994, Parrott et al. 1994, Zachow et al. 1997, Osuga et al. 1999, Ito et al. 2001, Shimizu et al. 2003), some controversy exists regarding the cellular origin of 
intraovarian HGF and c-met. It has been shown that HGF is secreted by theca cells (Parrott et al. 1994); whereas c-met is expressed within GC (Parrott et al. 1994, Zachow et al. 2000). This model fits the general pattern of mesenchymal HGF targeting epithelial c-met that has been documented in other tissues (Gheradi \& Stoker 1991). In this context, the effects of theca cell-derived HGF would then be predicted to be exclusive within GC. However, HGF can regulate rat theca cell steroidogenesis in vitro (Zachow et al. 1997), and this would suggest that theca cells contain c-met. In fact, theca cells and GC express HGF mRNA (Zachow et al. 1997); therefore, we propose a broader function for intraovarian HGF. Importantly, this more elaborate pattern for the expression of follicular HGF and c-met has been corroborated in other species (Parrott et al. 1994, Zachow et al. 1997, 2000, Osuga et al. 1999, Ito et al. 2001, Shimizu et al. 2003). Although some question does exist regarding which follicular cells produce HGF and c-met proteins, there is precedent for the expression of HGF and c-met by both theca cells and GC.

A third component of the HGF system, HGF activator (HGFA) protein, is a serine protease that cleaves the immature HGF precursor molecule to yield mature bioactive HGF (Miyazawa et al. 1993). The presence of ovarian HGFA has not been previously reported; however, HGFA represents a potential control mechanism for regulating local bioactive HGF concentrations.

In order to better define the role of HGF during folliculogenesis, this study investigated the effect of HGF on apoptosis in cultures of primary rat GC and isolated follicles. To complement this, the cellular distribution of the ovarian HGF system (HGF, c-met, and HGFA) during rat folliculogenesis was also evaluated.

\section{Materials and Methods}

\section{Apoptosis study: GC and follicle culture}

The usage of rats received institutional approval from RWJMS-UMDNJ and was conducted under $\mathrm{NIH}$ approved guidelines. Non-hormonally primed, intact, immature (23-25-day-old) Sprague-Dawley rats (Hilltop, Scottdale, PA, USA) were euthanized via $\mathrm{CO}_{2}$ inhalation. Ovaries were removed and placed in ice-cold Medium 199 (M199, Gibco BRL) that was supplemented with penicillin-streptomycin sulfate and $10 \%$ fetal bovine serum (FBS, Gibco BRL).

GCs were harvested in M199 using follicle puncture. Collected GC were centrifuged ( $5 \mathrm{~min}, 200 \mathrm{~g}, 4^{\circ} \mathrm{C}$ ), and resuspended in Dulbecco's modified Eagle's medium (DMEM, Gibco BRL) containing antibiotics and 5\% FBS. Cell viability and number were determined via the Trypan Blue exclusion test, using a hemacytometer.
Intact preantral follicles were isolated from rat ovaries by microdissection.

Upon isolation, $5 \times 10^{5}$ viable GC/well (6-well plates, Falcon Primaria) or follicles (5-6/well, 24-well plates, Falcon Primaria) were incubated at $37^{\circ} \mathrm{C}$, in a humidified atmosphere containing $5 \% \mathrm{CO}_{2}$ in air. Cultures were incubated for the first $24 \mathrm{~h}$ in DMEM containing 5\% FBS; then media was changed to serumfree, and hormones were added as described later.

\section{Detection of apoptotic GC}

Following serum withdrawal, follicles, and GC were incubated in the presence and absence of HGF (rhHGF, R\&D Systems, Minneapolis, MN, USA; 30, 60, and $90 \mathrm{ng} / \mathrm{ml}$ ). Following the addition of HGF, follicle, and GC cultures were incubated for $24 \mathrm{~h}$, and at $24 \mathrm{~h}$, YO-PRO-1 (final concentration $=0.1 \mu \mathrm{M}$; Molecular Probes, Inc., Eugene, OR, USA) was added to the wells based upon the method described by Peluso et al. (2001). In brief, a loss in plasma membrane integrity enables YO-PRO-1 to enter apoptotic cells, while healthy cells remain impermeable to this nucleic acid stain. After $10 \mathrm{~min}$ in the presence of YO-PRO- $1\left(37^{\circ} \mathrm{C}\right)$, cultures were examined via fluorescence microscopy $(200 \times)$ using a fluorescein isothiocyanate (FITC) filter set. For isolated GC, separate fields were evaluated in each treatment group so that 300 GCs were examined for quantitative analysis. For follicle cultures, a minimum of three size-matched follicles was compared for each treatment.

\section{Immunohistochemistry}

Twenty-three-day-old Sprague-Dawley rats were injected with either equine chorionic gonadotropin (eCG) (15 IU, Sigma) to induce the growth of large antral follicles, or vehicle (normal saline). Five nonprimed and five eCG-primed rats (ten ovaries for each) were used in three experiments. Forty-eight hours after the injections, rats were euthanized as described previously. Ovaries were removed and placed in ice-cold M199 that was supplemented with $10 \%$ FBS before being fixed in Bouin's fluid for $4 \mathrm{~h}$ (room temperature). Following the incubation in Bouin's fluid, ovaries were washed approximately ten times in $70 \%$ ethanol, and then stored in $70 \%$ ethanol $\left(4{ }^{\circ} \mathrm{C}\right)$ pending immunohistochemistry (Uzumcu et al. 2000).

Fixed ovarian tissue was embedded in paraffin and then sectioned at 4-5 $\mu \mathrm{m}$. The sections were deparaffinized, rehydrated, and heated (using a microwave oven) to boiling $0.01 \mathrm{M}$ citrate buffer ( $\mathrm{pH}$ 6.0). Upon boiling, the sections were subsequently heated (low heat setting) for an additional $15 \mathrm{~min}$. The sections were then blocked in $1.5 \%$ normal serum for $10 \mathrm{~min}$ at room temperature. 
The following antisera (Santa Cruz Biotechnology, Inc., Santa Cruz, CA, USA) were used: HGF (sc-7949), c-met (sc-162), and HGFA (sc-1371).

The ovarian sections were incubated with antisera overnight at room temperature using the following dilutions for anti-HGF, anti-c-met, and anti-HGFA antisera respectively: 1:50, 1:200, 1:200. Immunoreactivity (as identified by brown color) to the respective protein was then visualized using the $A B C$ Staining System (Santa Cruz Biotechnology) following the manufacturer's protocol. The negative control sections for HGF, c-met, or HGFA were incubated with normal rabbit or goat $\lg \mathrm{G}$ instead of primary antibody. Additional negative control sections for c-met and HGFA were incubated with c-met and HGFA primary antibodies that were preadsorbed with ten times c-met and HGFA-blocking peptides (Santa Cruz Biotechnology) respectively prior to conducting the detection protocol.

Sections were counterstained with Mayer's hematoxylin. The sections were then photographed using a Nikon imaging station equipped with a Nikon Eclipse E800 light microscope and Nikon DXM1200F digital camera.

\section{Scoring of the relative immunohistochemical staining intensity}

Differences in staining intensity were evaluated using a subjective scoring system from 0 to +4 , with a half-point increments $(0$, no staining and +4 , the strongest staining). Immunostaining intensities from two entire serial sections from all ovaries were evaluated for each antibody. The total score was divided by the number of sections, in which a respective structure (e.g., mid/large antral follicles, blood vessels) was observed.

\section{Western-blot analysis}

To determine the specificity of the antibodies used in immunohistochemistry, western-blot analysis was performed as described previously (Uzumcu et al. 2002). Briefly, harvested immature rat ovaries were lysed with $1 \times$ cell lysis buffer (Cell Signaling Technology, Beverly, MA, USA). The protein concentrations within the supernatants were measured using the DC Protein Assay Kit II (BioRad). Approximately, $50 \mu \mathrm{g}$ protein in sample-loading buffer was boiled for $5 \mathrm{~min}$ and then loaded into $10 \%$ (HGF and c-met) or 15\% (HGFA) SDS gels. Upon transfer onto an Immobilon $\mathrm{P}$ membranes (Millipore, Bedford, MA, USA), proteins were probed with the specific antibodies that were used for HGF, c-met, or HGFA IHC as described previously (Santa Cruz Biotechnology). Following incubation with a secondary antibody, the specific antigen-antibody complex was visualized using ECL Advance Western Blotting Detection Kit (GE Biosciences, Piscataway, NJ, USA) and viewed by autoradiography. Molecular weights were estimated by comparing visible bands against Kaleidoscope Prestained Standards (cat. \#161-0324; BioRad).

\section{Statistical analyses}

All experiments were repeated three times. For GC counts, mean values from independent experiments were statistically analyzed using one-way ANOVA followed by Tukey's test. Values were determined to be significant when $P<0.05$.

\section{Results}

\section{The effect of HGF on apoptosis in isolated GC and intact follicles}

Fluorescence was apparent in the presence and absence (control) of HGF. However, when compared to cultures

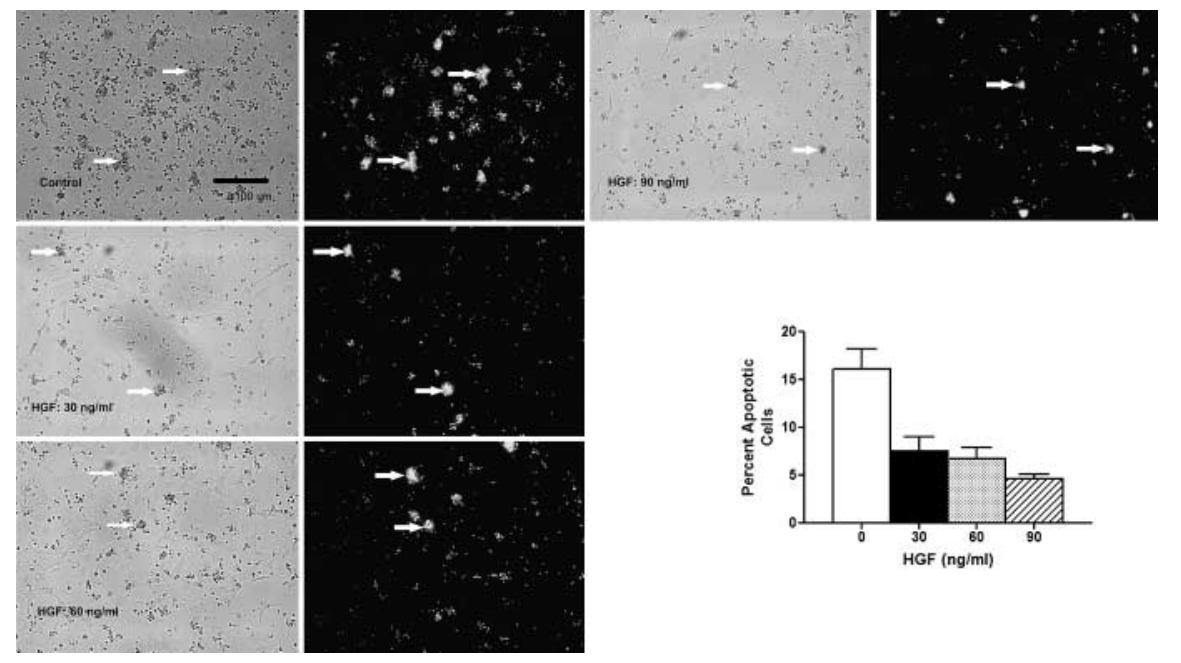

Figure 1 The effect of hepatocyte growth factor (HGF) on apoptosis in isolated rat granulosa cell (GC). GCs were given the HGF doses shown on the panels. Control GCs were untreated. YO-PRO-1 fluorescence was used to identify apoptotic GC as described in Materials and Methods. Paired brightfield and fluorescein isothiocyanate (FITC) images are shown for each treatment, and are representative from independent experiments. Arrows indicate examples of apoptotic GC in brightfield and FITC images (original magnification, 200×). Bar chart: Cell counts were performed to quantify percent apoptotic GC. Values shown represent means \pm S.E.M.; $* P<0.05$. 
that received HGF, YO-PRO-1-induced fluorescence was more profound in control GC (Fig. 1, arrows). This elevated level of fluorescence is indicative of a higher incidence of apoptosis in these cultures (Peluso et al. 2001). Counting of brightly fluorescent GC showed that when compared to control GC, HGF induced a statistically significant $(P<0.05)$ reduction in the number of apoptotic GC (Fig. 1, bar chart).

In isolated follicles, fluorescence also occurred in the presence and absence of HGF. Similar to what was

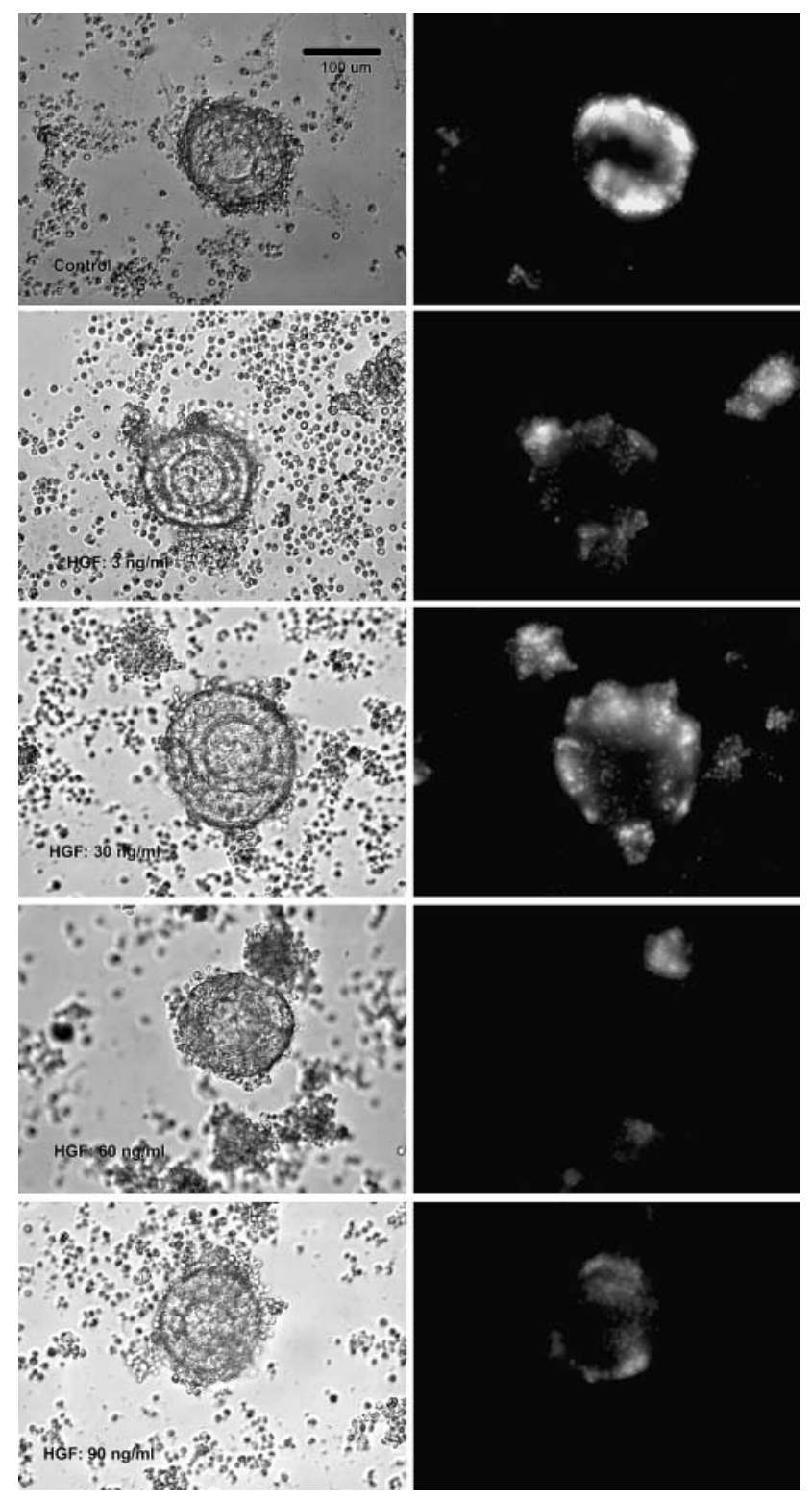

Figure 2 The effect of HGF on apoptosis in isolated preantral rat follicles. Follicles were given HGF doses as shown on the panels. Control follicles were untreated. YO-PRO-1 fluorescence was used to identify apoptotic cells as described in Material and Methods. Paired brightfield and FITC images are shown for each treatment, and are representative from independent experiments (original magnification, $200 \times$ ). observed in isolated GC, when compared to control (untreated) follicles, the level and intensity of fluorescence within follicular GC was markedly reduced in the presence of HGF (Fig. 2). This effect was most apparent with the higher doses of HGF (60 and $90 \mathrm{ng} / \mathrm{ml}$ ) that were given.

\section{Localization of immunoreactive HGF, c-met, and HGFA}

\section{HGF}

In non-primed ovaries, immunoreactive HGF was observed in GC within some preantral follicles at various stages of development (Fig. 3a and c, Table 1). Select GC within antral follicles also contained immunoreactive HGF protein. In contrast, a relatively low HGF signal was apparent within some theca cells in non-primed ovaries.

In eCG-primed ovaries, marked HGF staining was seen in GC within the majority of the preantral follicles; furthermore, distinct GC within some of these follicles demonstrated intense levels of immunoreactive HGF (Fig. 3b and d). Populations of GC within large antral follicles showed a muted signal for HGF; however, a general shift in staining from the GC to the thecainterstitial cell layers occurred following exposure to eCG (Fig. 3b, d and f, Table 1). The negative control sections showed no immunoreactivity for HGF (Fig. 3e).

c-met

In non-primed ovaries, staining was detected in GC within, and theca cells surrounding, preantral follicles (Fig. 4a and c, Table 1). Some intense levels of immunoreactive c-met were present in GC within certain small preantral follicles, as well as distinct GC within antral follicles (Fig. 4c). Within the c-met-positive preantral follicles, the level of immunoreactive c-met that was observed in the GC was arguably greater than that detected in theca cells. Structures resembling blood vessels within interstitium also showed immunoreactive c-met (Fig. 4a).

In eCG-primed ovaries, less intense staining for c-met was observed in GC within the smallest preantral follicles (Fig. 4b) when compared to that detected in size-matched follicles within non-primed ovaries (Fig. 4a). Moreover, there were no noticeably exclusive populations of intensely stained GC within large antral follicles, in contrast to what was observed in non-primed ovaries. In large antral follicles, immunoreactive c-met was seen in the theca cells and regions of the interstitium, concomitant with a virtual absence of staining within the GC (Fig. 4d, Table 1). The negative control sections showed no or minimal immunoreactivity (Fig. 4e).

\section{HGFA}

In non-primed ovaries, a somewhat uniform HGFA signal was observed in GC within, and theca cells surrounding, preantral follicles (Fig. 5a and c, Table 1). 

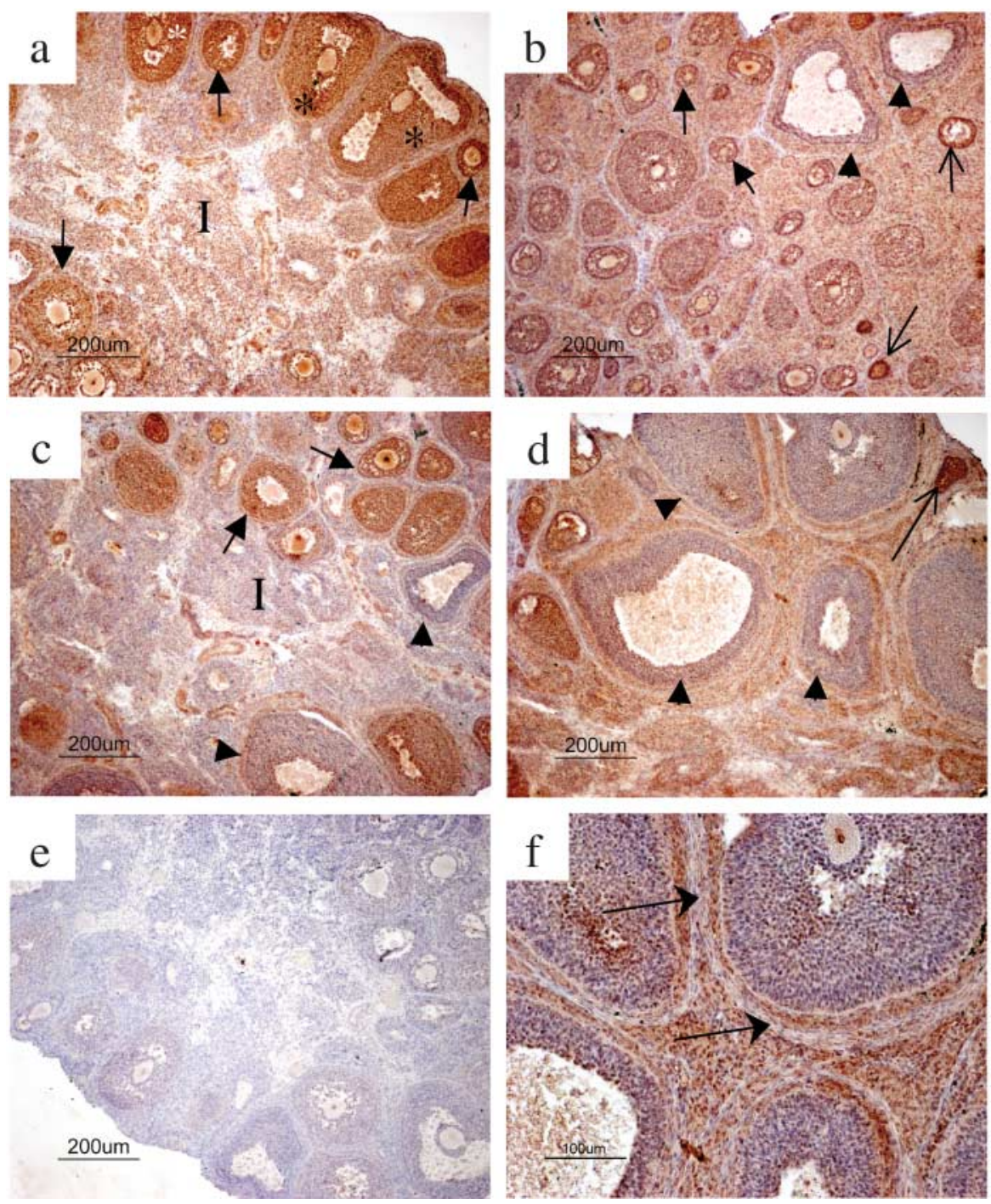

Figure 3 Immunolocalization of HGF within the rat ovary. (a) and (c) Immunohistochemistry was performed as described in Materials and Methods. Immunoreactivity (brown color) to HGF was observed in GC in preantral (arrows), as well as early antral (asterisks) follicles in non-primed ovaries as shown in a representative micrograph. The GC within some large antral follicles had no staining (arrowheads). The immunoreactivity was much lower in theca cells and non-existent in the interstitium (I) in non-primed ovaries. In equine chorionic gonadotropin (eCG)-primed ovaries, the immunoreactivity in GC was reduced in preantral follicles (arrows) and was shifted from GC to theca cells in antral follicles (arrowheads) as shown in a representative micrograph ( $b$ and $d)$. Certain secondary small preantral follicles showed strong staining (open arrows). (f) The theca externa surrounding large antral follicles (stealth arrows) was not stained. (e) Negative control sections showed no HGF immunoreactivity for which a typical example is shown. The data represent ten ovaries from five animals in three independent experiments.

The same general pattern of immunoreactive HGFA was apparent within the antral follicles that were present within these ovaries (Fig. 5a). Relatively low signal for HGFA was detected within the interstitium.

In eCG-primed ovaries, a more intense signal for HGFA was present in theca cells when compared to that detected within GC (Fig. 5b and d, Table 1). This pattern for HGFA was especially apparent in the theca cells associated with large antral follicles (Fig. 5d and f). The GC within large antral follicles showed no immunoreactive HGFA (Fig. 5f). The theca externa and the interstitium were devoid of HGFA immunoreactivity
(Fig. 5f, Table 1). Immunoreactive HGFA was not detected in the negative control sections (Fig. 5e).

Western analysis was used to verify the specificity of the antisera used in this study. As shown in Fig. 6, a major bands corresponding HGF, c-met, and HGFA were detected ( $\mathrm{A}-\mathrm{C}$ respectively).

\section{Discussion}

This study demonstrated that HGF can reduce the onset of apoptosis in rat GC in vitro. The anti-apoptotic effects of HGF were observed in both primary cultures of GC 
Table 1 Staining intensity of various ovarian structures for HGF, c-met, and HGFA in non-primed (control) and eCG-primed (eCG) ovaries.

\begin{tabular}{|c|c|c|c|c|c|c|}
\hline & \multicolumn{3}{|c|}{ Control } & \multicolumn{3}{|c|}{ eCG } \\
\hline & HGF & c-met & HGFA & HGF & c-met & HGFA \\
\hline Preantral GC & 2.8 & 2.4 & 2.4 & 2.6 & 1.8 & 1.9 \\
\hline Preantral TC & 1.6 & 1.8 & 1.9 & 2 & 1.9 & 1.5 \\
\hline Early antral GC & 3.3 & 2.6 & 2.4 & 2.3 & 1.3 & 1.7 \\
\hline Early antral TC & 1.6 & 2.4 & 2.4 & 2.8 & 3.3 & 3.2 \\
\hline $\mathrm{Mid} /$ large antral GC & 1.2 & 0.9 & 0.8 & 1.5 & 1 & 1.5 \\
\hline Mid/large antral TC & 2.2 & 1.8 & 1.7 & 3.5 & 3.7 & 3.7 \\
\hline $\begin{array}{l}\text { Theca externa (interstitium immediately } \\
\text { surrounding follicles) }\end{array}$ & 0.5 & 0 & 0 & 0.5 & 0 & 0 \\
\hline Interstitium (others) & 0.7 & 0.8 & 1 & 2 & 2 & 2 \\
\hline Connective tissue & 0 & 0 & 0 & 0 & 0 & 0 \\
\hline Endothelium & 1 & 4 & 0 & 1 & 4 & 0 \\
\hline Surface epithelium (SE) & 2 & 1 & 1 & 2 & 1 & 1 \\
\hline SE mesenchyme & 1 & 0 & 0 & 1 & 0 & 0 \\
\hline
\end{tabular}

GC, granulosa cells; TC, theca cells; 0 , no staining; 4, most intense staining.

and GC within isolated preantral follicles harvested from immature rats. The anti-apoptotic effect of HGF in vitro was complemented by the observation that growing follicles contain immunoreactive HGF, c-met, and HGFA. Therefore, it is plausible that since growing follicles contain (and/or are enveloped by) a complete HGF system, HGF supports folliculogenesis by blocking apoptosis.

These new data extend those from previous studies, which have shown that HGF modulates theca cell and GC steroidogenesis in vitro (Zachow et al. 1997, Parrott \& Skinner 1998a). As reported using cultured immature rat theca cells, HGF dampens luteinizing hormone (LH)stimulated androgen production (Zachow et al. 1997); and in immature rat GC, HGF suppresses FSH-directed estrogen synthesis in vitro (Parrott \& Skinner, 1998a, Zachow et al. 2000). The downregulatory effects of HGF on ovarian steroidogenesis have implications with regard to guiding preantral folliculogenesis and selection of the dominant follicle. It can be suggested that by impairing androgen and estrogen secretion in younger follicles, HGF would block an abnormal accumulation of intrafollicular steroids that could lead to follicle atresia (Hutz et al. 1990, Billig et al. 1993, Hillier \& Tetsuka 1997). We would propose this steroid-modulatory action is one mechanism by which HGF could impede the induction of apoptosis in GC in vivo. On the other hand, the culture conditions that were used in the present study to investigate the effect of HGF on apoptosis did not include the addition of exogenous gonadotropins and/or steroid hormones. Therefore, it can be argued that an accumulation of androgens and/or estrogens within the culture microenvironment did not instigate the onset of apoptosis in GC. Rather, in the present study, it is likely that HGF exerted a direct anti-apoptotic effect within GC. Future studies will be required in order to investigate the intracellular mechanisms of action that are affected by HGF in this context. Certainly, the effects of HGF within the so-called survival signaling cascades (e.g., those mediated by the Bcl family of proteins) would be useful in more thoroughly modeling the molecular mechanisms by which HGF suppresses apoptosis in GC.

Prior reports have shown that HGF and c-met are expressed within the ovary. For example, HGF has been measured in human follicular fluid; whereas the mRNAs for both HGF and c-met were detected in isolated human GC (Osuga et al. 1999). In the cow, HGF and c-met were measured in isolated theca cells and GC respectively (Parrott et al. 1994). In the pig, Shimizu et al. showed that theca cells expressed c-met, with HGF transcript expressed in GC Shimizu et al. (2003). Finally, in the rat, HGF mRNA was measured in both theca cells and GC (Zachow et al. 1997), with hormone-dependent changes in the relative level of c-met mRNA reported in GC (Zachow et al. 2000). To our knowledge, prior reports have not, however, described changes in the relative abundance of HGF and c-met proteins during in vivo folliculogenesis. Using freshly isolated bovine follicles, Parrott et al. have showed that the largest follicles contained the greatest abundance of c-met and HGF mRNAs (Parrott \& Skinner, 1998a); although, comparison of the specific cellular origin (i.e., theca cells vs GC) for HGF and c-met transcripts was not reported. In another study, hCG was shown to stimulate HGF mRNA expression in bovine theca cells in vitro (Parrott \& Skinner, 1998a). Interestingly, the present data showed that eCG priming induced a shift in the location of immunoreactive HGF from predominantly GC in nonprimed rats, to theca cells following the administration of eCG. Therefore, it can be proposed that the production of HGF within theca cells is stimulated by gonadotropins; however, the physiologic implications for this change in HGF content are uncertain.

It has previously been shown that both rat GC and theca cells respond to HGF stimulation in vitro (Zachow et al. 1997, 2000); and although Zachow et al. described the presence of theca cell c-met mRNA Zachow et al. (1997), this finding has been challenged to suggest that 

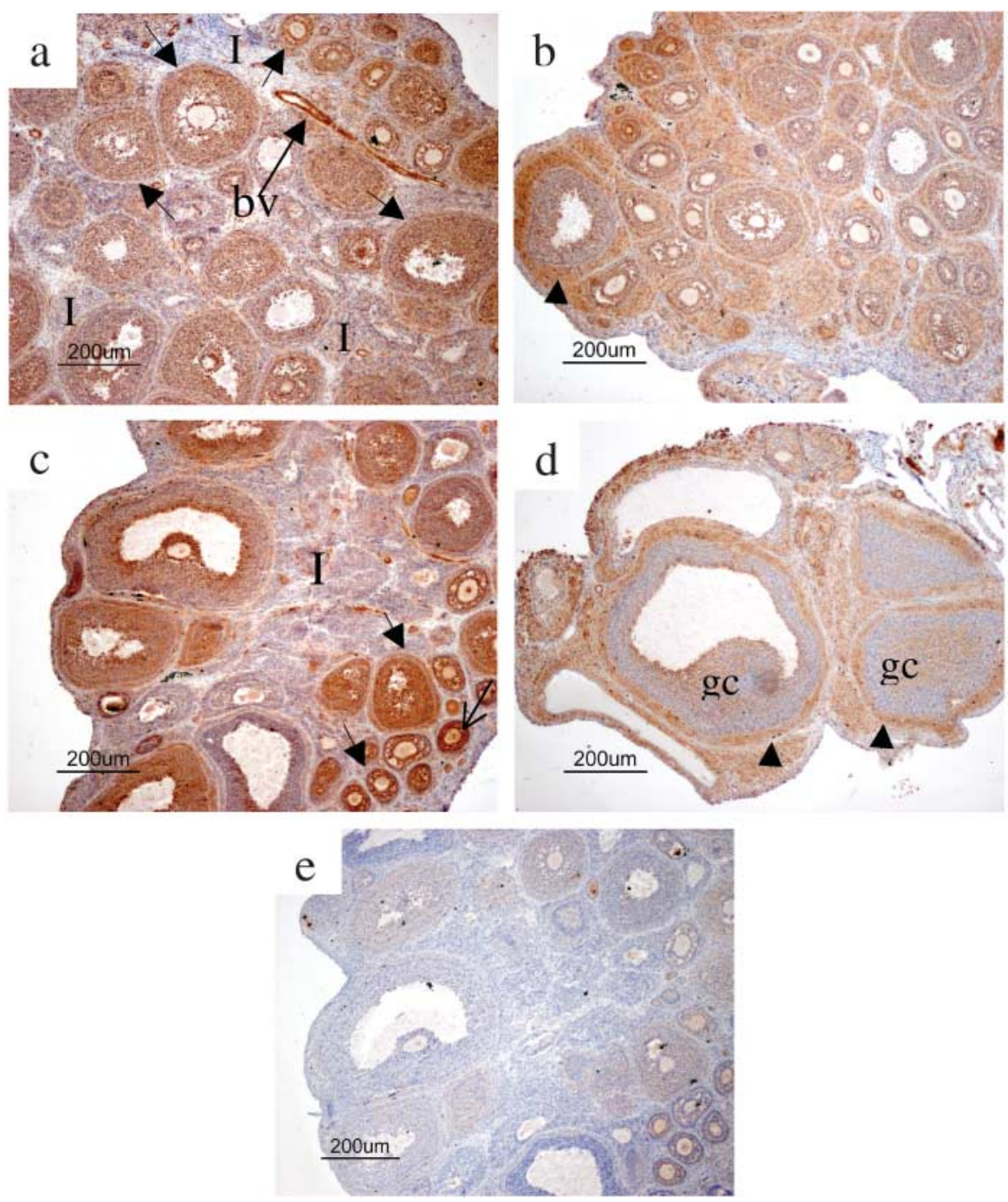

Figure 4 Immunolocalization of c-met within the rat ovary. (a) and (c) The immunoreactivity for c-met was observed in both in GC and theca cells in preantral and early antral follicles (arrows) in non-primed ovaries as shown in a representative micrograph. The interstitium (I) was generally negative for c-met in non-primed ovaries. Structures, presumably blood vessels, within the interstitium showed c-met immunoreactivity (bv). In primed ovaries, a stronger immunoreactivity for c-met was observed in theca cells when compared to GC in most follicles; this pattern was most apparent in large antral follicles (arrowheads; b and d). (d) Most GCs (gc) in large antral follicles were unstained. (e) Negative control showed minimal c-met immunoreactivity for which a typical example is shown. The data represent ten ovaries from five animals in three independent experiments.

the effects of HGF in theca cells were actually a phenomenon mediated by undefined factors produced within the theca cell cultures (Lail-Trecker et al. 1998). The present data do, however, show that theca cells contain c-met protein and this extends the previous data showing that these cells express c-met mRNA (Zachow et al. 1997). Interestingly, in porcine theca cells, the relative abundance of $\mathrm{c}$-met mRNA did not change following in vivo treatment with eCG (Shimizu et al. 2003), and LH did not induce changes in the amount of c-met mRNA in rat theca cells in vitro (Zachow et al. 1997). These data contrast with the observations in the present study in which eCG induced an increase in the presence of immunoreactive c-met in the rat theca cells in vivo. On the other hand, in primary rat GC cultures, c-met expression was reduced by FSH (Zachow et al. 2000), and as shown in this report, eCG caused a noticeable decrease in immunoreactive c-met in rat GC in vivo. Thus, data from independent investigations, using both in vivo and in vitro approaches, show that c-met content within GC can be reduced by gonadotropin stimulation. These findings support a model in which the effects of HGF within preovulatory GC can be controlled in response to hormonally mediated levels of c-met. Specifically, a gonadotropin-induced reduction in c-met within large antral follicles would likely impair follicular HGF bioactivity. Moreover, the present study is the first to show that immunoreactive HGFA is located within the ovary. Whether or not ovarian HGF bioactivity depends upon, or is modulated by, local HGFA is 

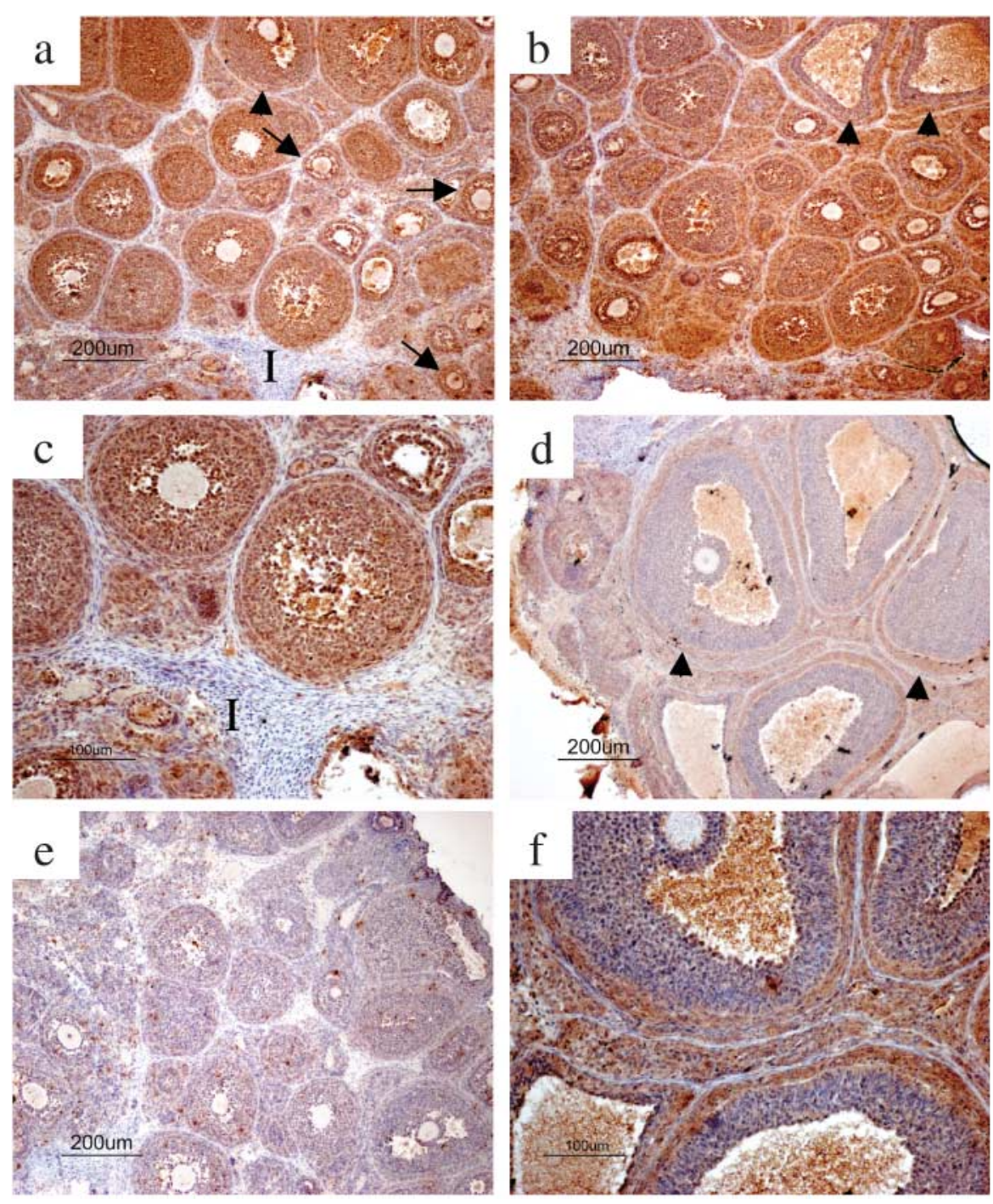

Figure 5 Immunolocalization of HGFA within the rat ovary. (a) and (c) The immunoreactivity for HGFA was observed at similar levels in GC and theca cells of preantral follicles (arrows) and early antral follicles (arrowhead) in non-primed ovaries. The interstitium (I) also showed little or no immunoreactivity to HGFA in non-primed ovaries (a and c). (b) In eCG-primed ovaries, preantral follicles demonstrated a relatively uniform level of HGFA within the GC and theca cells. (d) and (f) In large antral follicles, the staining intensity was higher in the theca layer, when compared to that detected in the GC within large antral follicles (arrowheads) in eCG-primed ovaries. The theca externa was unstained in both non-primed and primed ovaries, which is clearly shown in higher magnification micrographs in (c) and $(\mathrm{f})$ respectively. (e) The negative control sections showed no HGFA immunoreactivity for which a representative section is shown. Data represent ten ovaries from five animals in three independent experiments. unknown at this time. It is noteworthy, however, that eCG did cause a shift in the cellular distribution of ovarian HGFA. Therefore, like HGF and c-met, it appears that stage-specific changes in available HGFA occur during follicle development.

Based upon the data presented in this report, and those previously published by this Lab. and others, it is very likely that HGF exerts multiple levels of control over GC and theca cell function which collectively enable the progression of folliculogenesis. We suggest that a complete HGF system within preantral follicles (as identified in this study) down-modulates gonadotropinstimulated steroidogenesis, and concomitantly supports GC survival via an anti-apoptotic effect. The aforementioned would be complemented by the paracrine mitogenic effects of the previously described HGF-kit ligand system (Parrott \& Skinner 1998b). As antral follicle growth proceeds, gonadotropin-orchestrated changes in

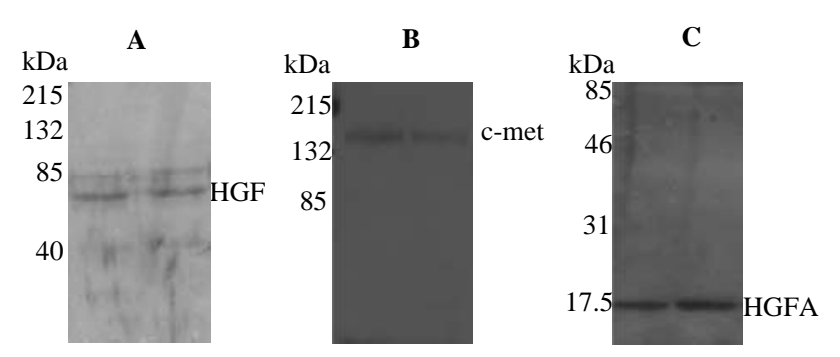

Figure 6 Western-blot analysis for HGF, c-met, and HGFA in immature rat ovaries. Western-blot 425 analysis was performed using whole ovarian lysate as described in the text. (A) The analysis shows a major band approximately at $69 \mathrm{kDa}$, the expected size for HGF $\alpha$. The minor second band above the major bind is likely to be glycosylated form of the HGFa. (B) Western analysis for c-met showed a single band at expected size (140 kDa). (C) A strong and single band was observed in HGFA western analysis at approximately $17.5 \mathrm{kDa}$. 
the distribution of HGF, c-met, and/or HGFA are likely to regulate HGF bioactivity, perhaps leading to the alleviation of the HGF-directed suppression of gonadotropin-stimulated androgen and estrogen production within the dominant follicle. It is thus enticing to predict that manipulation of the intraovarian HGF system may provide a novel approach to controlling the balance between follicular selection and atresia.

\section{Acknowledgements}

We gratefully acknowledge Dr John Peluso (Department of Cell Biology, University of Connecticut Health Center, Farmington, CT) for his conceptual input. Supported by NIEHS grant P30 ES05022-17 (MU), UMDNJ Foundation Grant (ZP), and funds from Robert Wood Johnson Medical School-UMDNJ (RZ).

\section{References}

Billig H, Furuta I \& Hsueh AJ 1993 Estrogens inhibit and androgens enhance ovarian granulosa cell apoptosis. Endocrinology 133 2204-2212.

Gheradi E \& Stoker M 1991 Hepatocyte growth factor-scatter factor: mitogen, motogen, and Met. Cancer Cells 3 227-232.

Hillier SG \& Tetsuka M 1997 Role of androgens in follicle maturation and atresia. Bailliere's Obstetrics and Gynecology 11 249-260.

Hussein MR 2005 Apoptosis in the ovary: molecular mechanisms. Human Reproduction Update 11 162-177.

Hutz RJ, Dierschke DJ \& Wolf RC 1990 Role of estradiol in regulating ovarian follicular atresia in rhesus monkeys: a review. Journal of Medical Primatology 19 553-571.

Ito M, Harada T, Tanikawa M, Fujii A, Shiota G \& Terakawa N 2001 Hepatocyte growth factor and stem cell factor involvement in paracrine interplays of theca and granulosa cells in the human ovary. Fertility and Sterility 75 973-979.

Lail-Trecker M, Gulati R \& Peluso JJ 1998 A role for hepatocyte growth factor/scatter factor in regulating normal and neoplastic cells of reproductive tissues. Journal of the Society for Gynecologic Investigation 5 114-121.

Liu Y, Lin L \& Zarnegar R 1994 Modulation of hepatocyte growth factor gene expression by estrogen in mouse ovary. Molecular and Cellular Endocrinology 104 173-181.

Miyazawa K, Shimomura T, Kitamura A, Kondo J, Morimoto Y \& Kitamura N 1993 Molecular cloning and sequence analysis of the cDNA for a human serine protease responsible for activation of hepatocyte growth factor, structural similarity of the protease precursor to blood coagulation factor-xii. The Journal of Biological Chemistry 269 10024-10028.
Osuga Y, Tsutsumi O, Momoeda M, Okagaki R, Matsumi H, Hiroi H, Suenaga A, Yano T \& Taketani Y 1999 Evidence for the presence of hepatocyte growth factor expression in human ovarian follicles. Molecular Human Reproduction 5 703-707.

Parrott JA, Vigne JL, Chu BZ \& Skinner MK 1994 Mesenchymal epithelial interactions within the ovarian follicle involve keratinocyte and hepatocyte growth factor production by theca cells and their action on granulosa cells. Endocrinology 135 569-575.

Parrott JA \& Skinner MK 1998a Developmental and hormonal regulation of hepatocyte growth factor expression and action in the bovine ovarian follicle. Biology of Reproduction $\mathbf{5 9}$ 553-560.

Parrott JA \& Skinner MK $1998 b$ Theca cell-granulosa cell interactions involve a positive feedback loop among keratinocyte growth factor, hepatocyte growth factor, and kit ligand during ovarian follicular development. Endocrinology 139 2240-2245.

Peluso JJ, Pappalardo A \& Fernandez G 2001 Basic fibroblast growth factor maintains calcium homeostasis and granulose cell viability by stimulating calcium efflux via a $\mathrm{PKC} \delta$-dependent pathway. Endocrinology 142 4203-4211.

Shimizu T, lijima K, Sasada H \& Sato E 2003 Messenger ribonucleic acid expressions of hepatocyte growth factor, angiopoietins and their receptors during follicular development in gilts. Journal of Reproduction and Development 49 203-211.

Uzumcu M, Homsi MF, Ball DK, Coskun S, Jarourdi K, Hollanders JM \& Brigstock DR 2000 Localization of connective tissue growth factor in human uterine tissues. Molecular Human Reproduction 6 10931098.

Uzumcu M, Westfall SD, Dirks KA \& Skinner MK 2002 Embryonic testis cord formation and mesonephric cell migration requires the phosphatidylinositol 3-kinase signaling pathway. Biology of Reproduction 67 1927-1935.

Zachow RJ, Weitsman SR \& Magoffin DA 1997 Hepatocyte growth factor regulates ovarian theca-interstitial cell differentiation and androgen production. Endocrinology 138 691-697.

Zachow RJ, Ramski BE \& Lee H 2000 Modulation of estrogen production and $17 \beta$-hydroxysteroid dehydrogenase-type 1, cytochrome P450 aromatase, c-Met, and protein kinase B $\alpha$ messenger ribonucleic content in rat ovarian granulosa cells by hepatocyte growth factor and follicle-stimulating hormone. Biology of Reproduction 62 1851-1857.

Zachow RJ \& Woolery JK 2002 The effect of hepatocyte growth factor on cyclic nucleotide-dependent signaling and steroidogenesis in rat granulosa cells in vitro. Biology of Reproduction 67 454-459.

Received 3 October 2005

First decision 14 November 2005

Revised manuscript received 17 April 2006

Accepted 16 May 2006 